\title{
Dynamics of Circulating miR-122 Predict Liver Cancer and Mortality in Japanese Patients with Histopathologically Confirmed NAFLD and Severe Fibrosis Stage
}

\author{
Norio Akuta ${ }^{\text {a }}$ Yusuke Kawamura $^{a}$ Fumitaka Suzuki $^{a}$ Satoshi Saitoh ${ }^{a}$ \\ Yasuji Arase $^{a}$ Nozomu Muraishi ${ }^{a}$ Shunichiro Fujiyama ${ }^{a}$ Hitomi Sezaki ${ }^{a}$ \\ Tetsuya Hosaka ${ }^{a}$ Masahiro Kobayashia ${ }^{a}$ Mariko Kobayashi ${ }^{b}$ Yoshiyuki Suzuki ${ }^{a}$ \\ Kenji Ikeda ${ }^{a}$ Hiromitsu Kumada ${ }^{a}$ \\ aDepartment of Hepatology, Toranomon Hospital and Okinaka Memorial Institute for Medical Research, Tokyo, \\ Japan; ' Liver Research Laboratory, Toranomon Hospital, Tokyo, Japan
}

\section{Keywords \\ Nonalcoholic fatty liver disease · Serial liver biopsy · microRNA-122 - Dynamics · Liver cancer · Mortality}

\begin{abstract}
Introduction: It is unclear whether the relationships between changes in fibrosis and circulating microRNA-122 (miR-122) dynamics might influence the prognosis of nonalcoholic fatty liver disease (NAFLD). Methods: This study investigates the impact of serum miR-122 dynamics and histological changes on the incidence of liver cancer and mortality in 81 Japanese NAFLD patients who underwent serial liver biopsies. The median interval between the first and second liver biopsies was 2.9 years. Results: The fibrosis stage scores indicated progression, no change, and improvement (a decrease of one point or more) in $21.0 \%, 56.8 \%$, and $22.2 \%$ of the patients, respectively. There were 64 patients in the high-risk group who had no improvement in stage scores. Among these, the miR-122 levels were significantly lower in 7 patients with liver cancer than those of the 54 patients who had no liver cancer at the second liver biopsy. The cumula-
\end{abstract}

karger@karger.com www.karger.com/ocl

Karger $\stackrel{\text { ' }}{5}$

BOPEN ACCESS
(C) 2021 The Author(s)

Published by S. Karger AG, Basel

This is an Open Access article licensed under the Creative Commons Attribution-NonCommercial-4.0 International License (CC BY-NC) (http://www.karger.com/Services/OpenAccessLicense), applicable to the online version of the article only. Usage and distribution for commercial purposes requires written permission. tive rates of liver cancer were significantly higher in cases with miR-122 ratios $<0.5$ (serum miR-122 level at second biopsy to that at first biopsy) than those with ratios $\geq 0.5$. The cumulative survival rates in cases with miR-122 ratios $<0.5$ tended to be lower than those with ratios $\geq 0.5$. Of the 64 high-risk patients, 39 indicated stage 2 or greater (severe fibrosis stage) at the first liver biopsy and also showed similar results of cumulative liver cancer and survival rates. Conclusions: Longitudinal examination of serial liver biopsies indicated that the circulating miR-122 dynamics might be useful in predicting the prognosis for NAFLD patients with severe fibrosis stage and no improvement of the stage scores.

(C) 2021 The Author(s).

Published by S. Karger AG, Basel

\section{Introduction}

Worldwide, the most common liver disease is nonalcoholic fatty liver disease (NAFLD) [1-6]. The liver pathology of this disease ranges from typically benign nonalcoholic fatty liver to nonalcoholic steatohepatitis 
(NASH), which may progress to liver cirrhosis, liver cancer, and finally liver failure [7]. Studies suggest that the fibrosis stage is a more reliable predictor of liver-specific mortality than the NAFLD activity score [8]. In contrast to other histopathological features of steatohepatitis, the fibrosis stage has been reported to be an independent and significant predictor of overall mortality, liver transplantation, and liver-related events [9].

Various environmental, genetic, and epigenetic factors are known to influence the development and progression of NAFLD. For example, the level of circulating microRNA-122 (miR-122), an epigenetic factor, has been associated with the histopathological severity of liver disease and mortality [10-12]. Our previous reports indicate that circulating miR-122 levels vary with the stage of liver cancer or NAFLD-related histopathological changes. In this context, a longitudinal follow-up study of 1 patient demonstrated a tendency for serum miR-122 levels to decrease before progression of the fibrosis stage and the development of liver cancer [13].

However, it is still unclear whether the relationships between fibrosis changes and circulating miR-122 dynamics might influence the prognosis of patients with NAFLD. Thus, the purpose of this retrospective study was to investigate whether circulating miR-122 dynamics might affect changes in the fibrosis stage, the development of liver cancer, and mortality. The study was based on the longitudinal examination of serial liver biopsies of 81 Japanese patients with NAFLD.

\section{Materials and Methods}

\section{Patients}

Between 1980 and 2021, there were 477 Japanese patients who were diagnosed with NAFLD based on histopathological examination of liver biopsies at Toranomon Hospital. At least 2 liver biopsies were performed on 95 of these patients, who were clinically evaluated in detail over time. The attending physician determined need for repeated liver biopsies.

Between the first and second biopsies, there were 81 of the 95 patients who were not exposed to drugs with high evidence levels for causing NAFLD, such as vitamin $\mathrm{E}$ and antidiabetic drugs (e.g., glucagon-like peptide- 1 receptor agonists, pioglitazone, and sodium-glucose transporter-2 inhibitors). Seventy-seven of 81 patients were diagnosed as NASH at the time of the first liver biopsy, according to the Fatty Liver Inhibition of Progression algorithm.

There was median time between biopsies of 2.9 years (range: $0.4-23.5$ years). In the time between biopsies, we examined the impact that serum miR-122 dynamics and histological changes have on the incidence of liver cancer and mortality among these 81 patients. Serial liver biopsies were performed on all patients (Table 1).

Based on the practice guidance from the American Association for the Study of Liver Diseases [14], the diagnosis of NAFLD was based on the liver histopathological findings of steatosis in $\geq 5 \%$ of hepatocytes after excluding other diseases of the liver (such as autoimmune hepatitis, primary biliary cholangitis, viral hepatitis, drug-induced liver disease, biliary obstruction, hemochromatosis, Wilson disease, and a-1-antitrypsin deficiency-associated liver disease). Of these 81 patients, there were none who consumed $>20$ $\mathrm{g}$ of alcohol per day.

The Human Ethics Review Committee at Toranomon Hospital approved the protocol of the study, and a signed informed consent form was obtained from each of the patients at the time of liver histological diagnosis. The study complied with the International Conference on Harmonization Guidelines for Good Clinical Practice (E6), as well as the 2013 Declaration of Helsinki.

\section{Liver Histopathology}

Steatosis grades $0,1,2$, and 3 were considered equivalent to hepatocyte steatosis levels of $<5 \%, 5-33 \%, 33-66 \%$, and $\geq 66 \%$, respectively. Lobular inflammation scores of $0,1,2$, and 3 were considered equivalent to a complete lack of foci, $<2$ foci, $2-4$ foci, and $\geq 4$ foci per $200 \times$ field, respectively. Hepatocyte ballooning scores of 0,1 , and 2 were considered equivalent to a complete lack of cells, few cells, and many cells, respectively. NAFLD activity score was considered as the sum of the steatosis, lobular inflammation, and hepatocyte ballooning scores, and its range was $0-8$ points [15]. The stages of fibrosis were defined as $0,1,2,3$, and $4[15,16]$.

At the time of the second biopsy, decreases of one or more points of the histopathological scores (relative to the first biopsy) were classified as "improvement," while increases of one or more points were considered as "progression." Patients were assigned to the high-risk group if they showed no improvement in the fibrosis stage scores, which are an important predictor of liver cancer and mortality $[8,9]$.

\section{Follow-Up}

After the NAFLD diagnosis, hematological and biochemical data were collected at least twice per year. At least once per year, patients underwent computed tomography, ultrasonography, or magnetic resonance imaging studies.

\section{Serum miR-122 Measurements}

Serum miR-122 levels were measured based on 2 previous reports $[13,17]$. The comparative cycle threshold method $\left(2^{-\Delta \Delta C T}\right)$ $[18,19]$ was used to calculate the relative expression of serum miR122 , and spiked cel-miR-39 served as a normalized internal control $[13,17]$. The serum miR-122 ratio was calculated as the ratio of the measurement at the second biopsy to that at the first biopsy.

\section{Statistical Analysis}

The characteristics of the groups were compared using the Mann-Whitney $U$ test, and the cumulative survival rates and $\mathrm{cu}-$ mulative incidence rates of liver cancer were calculated using the Kaplan-Meier technique. The log-rank test was used to analyze differences in curves between groups. The survival rate was analyzed for the time between the biopsy and death or the last visit, while the incidence rate of liver cancer was analyzed for the time between the biopsy and the occurrence of liver cancer or the last visit. $p$ values $<0.05$ according to a 2 -tailed test were used to determine significance. The software SPSS (SPSS Inc., Chicago, IL, USA) was used for the statistical comparisons. 
Table 1. Characteristics of 81 NAFLD patients at the first and second liver biopsies

\begin{tabular}{lll}
\hline & First biopsy & Second biopsy \\
\hline $\begin{array}{l}\text { Demographic data } \\
\quad \text { Numbers of patients }\end{array}$ & & \\
$\quad$ Gender, male/female, $n$ & 81 & 81 \\
$\quad$ Age, year* & $47 / 34$ & $47 / 34$ \\
$\quad$ Body mass index, $\mathrm{kg} / \mathrm{m}^{2 *}$ & $52(24-76)$ & $59(26-82)$ \\
Histological findings & $27.3(20.5-40.8)$ & $26.7(19.1-38.6)$ \\
$\quad$ Steatosis, 0/1/2/3, $n$ & & \\
Lobular inflammation, 0/1/2/3, $n$ & $0 / 25 / 37 / 19$ & $3 / 30 / 33 / 15$ \\
$\quad$ Ballooning, 0/1/2, $n$ & $5 / 39 / 31 / 6$ & $2 / 46 / 31 / 2$ \\
$\quad$ Stage, 0/1/2/3/4, $n$ & $3 / 42 / 36$ & $5 / 58 / 18$ \\
$\quad$ NAFLD activity score, $\leq 2 / 3,4 / \geq 5, n$ & $6 / 19 / 14 / 34 / 8$ & $0 / 31 / 9 / 33 / 8$ \\
Laboratory data* & $3 / 27 / 51$ & $7 / 35 / 39$ \\
$\quad$ Serum aspartate aminotransferase, U/L & & \\
Serum alanine aminotransferase, U/L & $59(19-198)$ & $42(15-270)$ \\
$\quad$ Platelet count, $\times 10^{3} / \mathrm{mm}^{3}$ & $88(20-312)$ & $54(8-312)$ \\
$\quad$ Fibrosis-4 index & $204(40-389)$ & $207(74-333)$ \\
$\quad$ Serum miR-122, fold change & $1.57(0.41-5.00)$ & $1.62(0.53-6.68)$ \\
\hline
\end{tabular}

NAFLD, nonalcoholic fatty liver disease; miR-122, microRNA-122. Data are number of patients, except those denoted by*, which represent the median (range) values.

\section{Results}

\section{Histopathological Changes}

Table 2 summarizes the distribution of histopathological scores at the first and second liver biopsies. The steatosis scores indicated progression, no change, and improvement in $17.3 \%, 49.4 \%$, and $33.3 \%$ of the 81 patients, respectively (Table 2 ). The median change per year for the entire group was 0.000 /year (range, $-2.393-0.899 /$ year). The lobular inflammation score indicated progression, no change, and improvement in $22.2 \%, 51.9 \%$, and $25.9 \%$ of the patients, respectively (Table 2), and the median change per year was 0.000 /year (range, -1.429-1.287/ year). Analysis of the ballooning score indicated that $11.1 \%, 54.3 \%$, and $34.6 \%$ of the patients showed progression, no change, and improvement, respectively (Table 2), and the median change per year was 0.000 /year (range, -2.393-0.668/year). The stage scores indicated progression, no change, and improvement in $21.0 \%$, $56.8 \%$, and $22.2 \%$ of the patients, respectively (Table 2 ), and the median change per year was $0.000 /$ year (range, $-2.684-0.985 /$ year).

\section{Cumulative Liver Cancer and Survival Rates}

According to Histological Findings

All 81 patients were subjected to analyses to determine the cumulative liver cancer and survival rates according to the histological findings at the first liver biopsy. During the median follow-up of 7.6 years (range, $0.6-30.4$ years) from the first liver biopsy, 9 (11.1\%) cases of liver cancer and $8(9.9 \%)$ deaths were recorded after the second liver biopsy. In cases of liver cancer, the median interval between the first liver biopsy and the incidence of liver cancer was 4.2 years (range, 0.7-23.7 years). In cases of death, the median interval between the first liver biopsy and death was 7.1 years (range, 2.0-31.8 years).

The cumulative rates of liver cancer were not different among the 3 groups of steatosis scores ( $p=0.455$; logrank test), the 4 groups of lobular inflammation scores ( $p$ $=0.376$; log-rank test), and the 3 groups of ballooning scores $(p=0.904 ;$ log-rank test). However, these rates were significantly different among the 5 groups of stage scores ( $p=0.007$; log-rank test), as shown in Figure 1 . The cumulative survival rates were not different among the 3 groups of steatosis scores ( $p=0.6834$; log-rank test), the 4 groups of lobular inflammation scores $(p=0.259$; logrank test), and the 3 groups of ballooning scores ( $p=$ 0.713; log-rank test). However, the 5 groups of stage scores showed significant differences in these rates $(p=$ 0.042; log-rank test). Among the 4 types of histological findings, the present results indicate that the stage score was the most important predictor of prognoses, including liver cancer and mortality. 
Table 2. Distribution of histological scores at the first and second liver biopsies

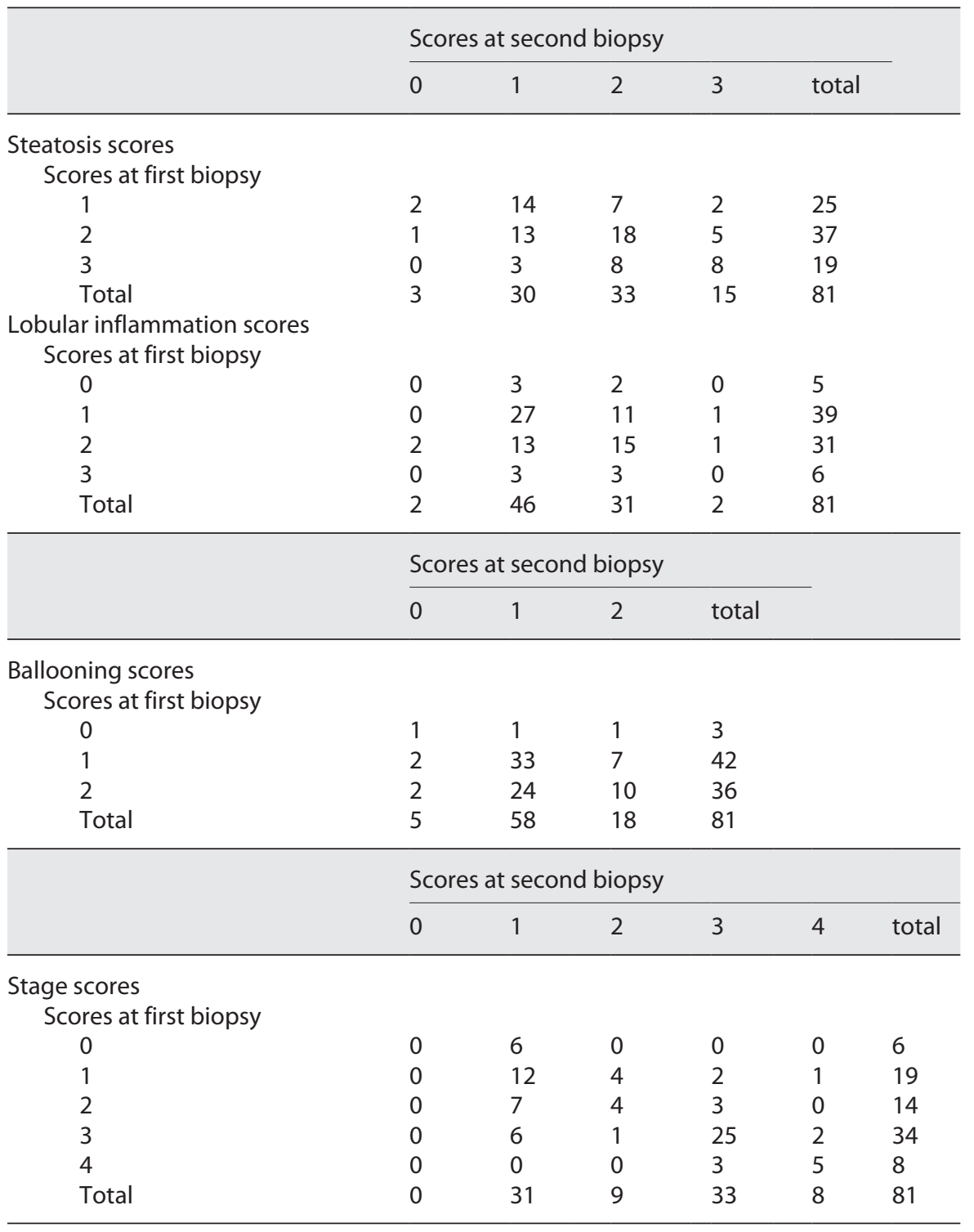

Development of Liver Cancer According to miR-122 Dynamics of High-Risk Group

Of the 81 patients, 64 did not show improvement of the stage scores and were considered as the high-risk group. These patients underwent analyses to determine the liver cancer development according to miR-122 dynamics. There were 7 patients (10.9\%) who developed liver cancer after the second liver biopsy. At the first liver biopsy, miR-122 levels of the 7 patients who developed liver cancer were not different from those of the 54 patients who did not develop it ( $p=0.851$; Mann-Whitney $\mathrm{U}$ test). At the second liver biopsy, the levels in patients with liver cancer were significantly lower than those in patients without liver cancer ( $p=0.015$; Mann-Whitney U test) (Fig. 2a).

Overall, the median miR ratio was 0.5 (range, 0.0-29). The group that did not develop liver cancer had a median miR-122 ratio of 0.6 (range, 0.0-29), and the group that did develop it had a median ratio of 0.2 (range, 0.1-2.1) (Fig. 2b). Thus, the cumulative survival rates and incidence rates of liver cancer were evaluated using a cutoff value of 0.5 for the median miR-122 ratio. The present results show a decrease of miR-122 levels at the second liver biopsy of the high-risk group without improvement of the stage scores, which suggests that the miR-122 dynamics is useful for prognosis prediction. 
Fig. 1. Cumulative rates of liver cancer according to fibrosis stage. The rates were significantly different among 5 stage scores $(p=0.007 ; \log$-rank test).

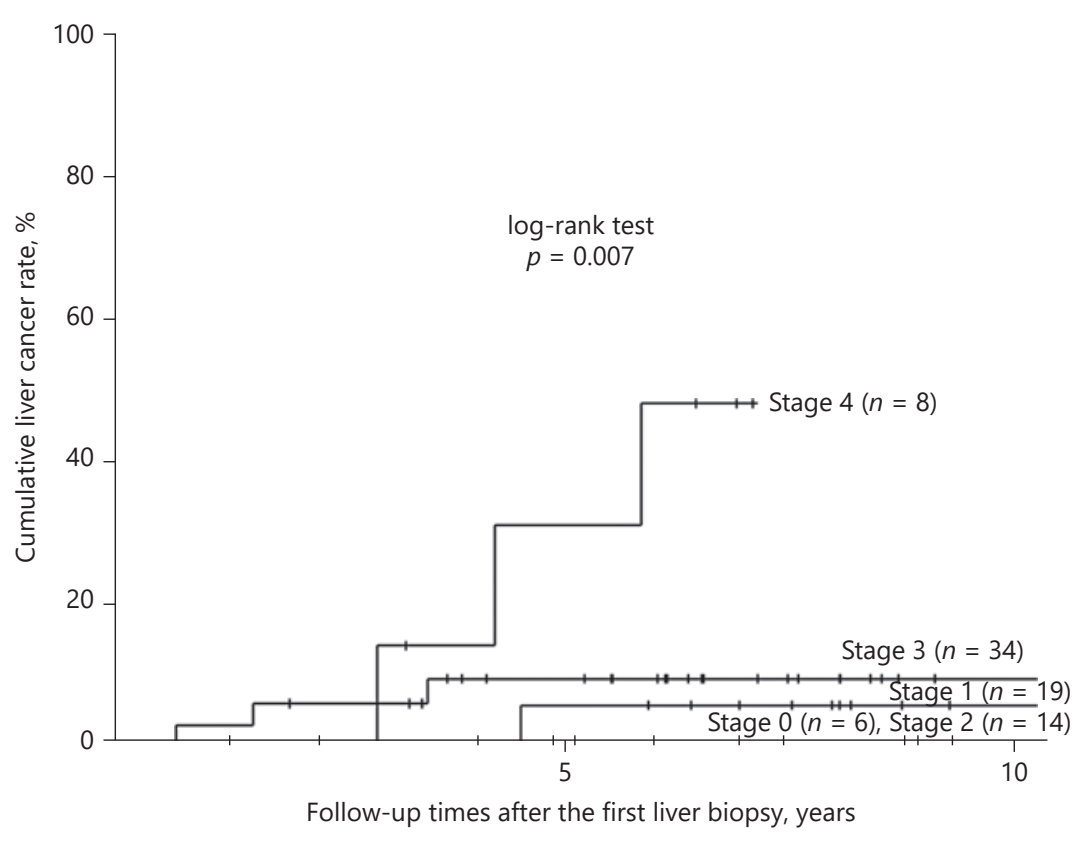

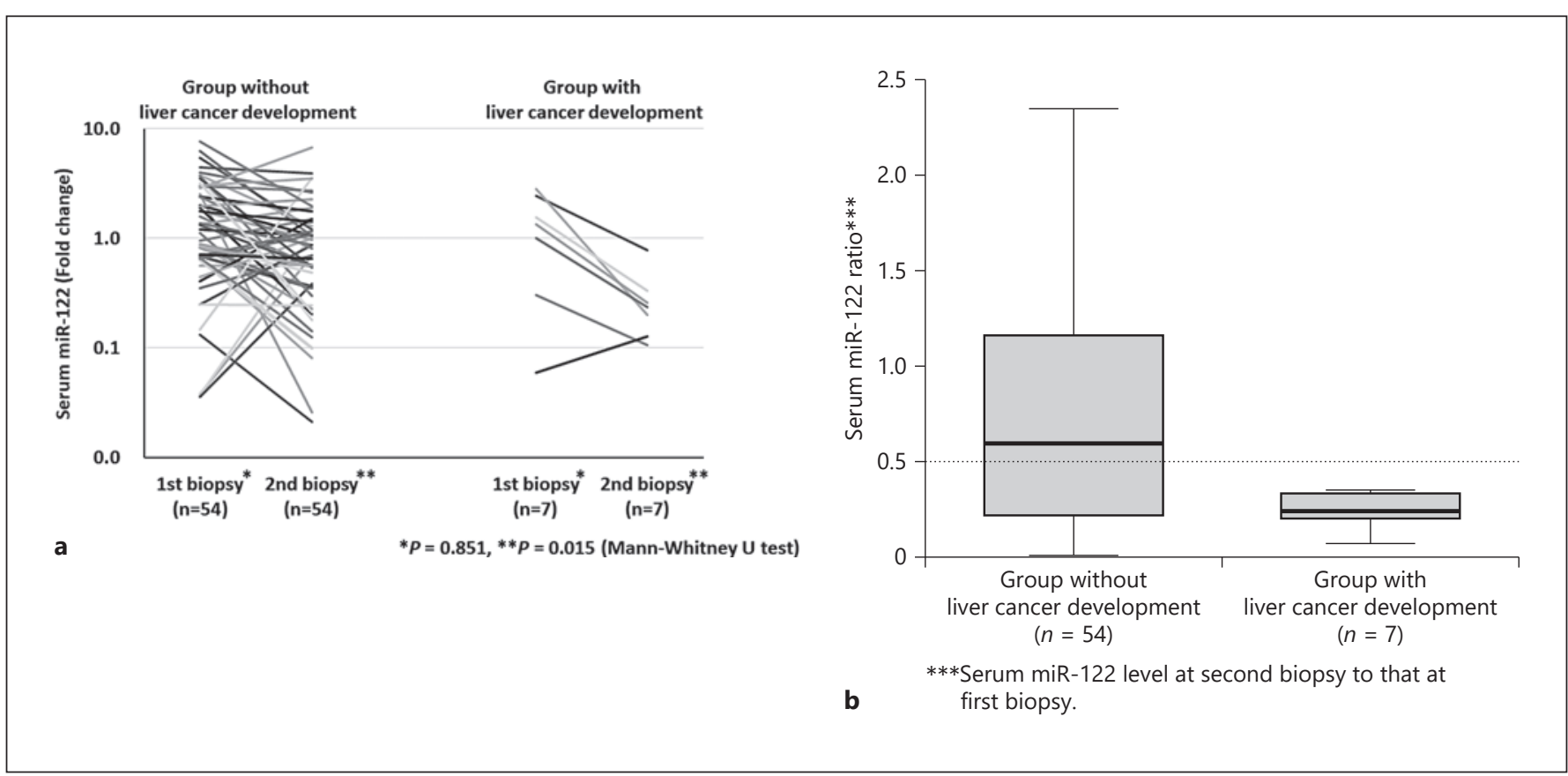

Fig. 2. Serum miR-122 dynamics according to whether liver cancer developed. a Logarithmically transformed levels of serum miR122 at the first and second liver biopsies. *At first liver biopsy, miR122 levels in patients who developed liver cancer were not different from those who did not develop liver cancer ( $p=0.851$; MannWhitney $U$ test). ${ }^{* *}$ At second liver biopsy, the levels in patients with liver cancer were significantly lower than those in patients without liver cancer ( $p=0.015$; Mann-Whitney U test). b Serum
miR-122 ratio at the first and second liver biopsies (ratio of serum miR-122 level at second biopsy to that at first biopsy). Bars within the boxes indicate the median value. The boxes denote the 25 th75th percentiles, and the lower and upper bars denote the 10th90th percentiles, respectively. The cumulative survival rates and incidence rates of liver cancer were evaluated using a cutoff value of 0.5 for the miR-122 ratio. miR-122, microRNA- 122 . 


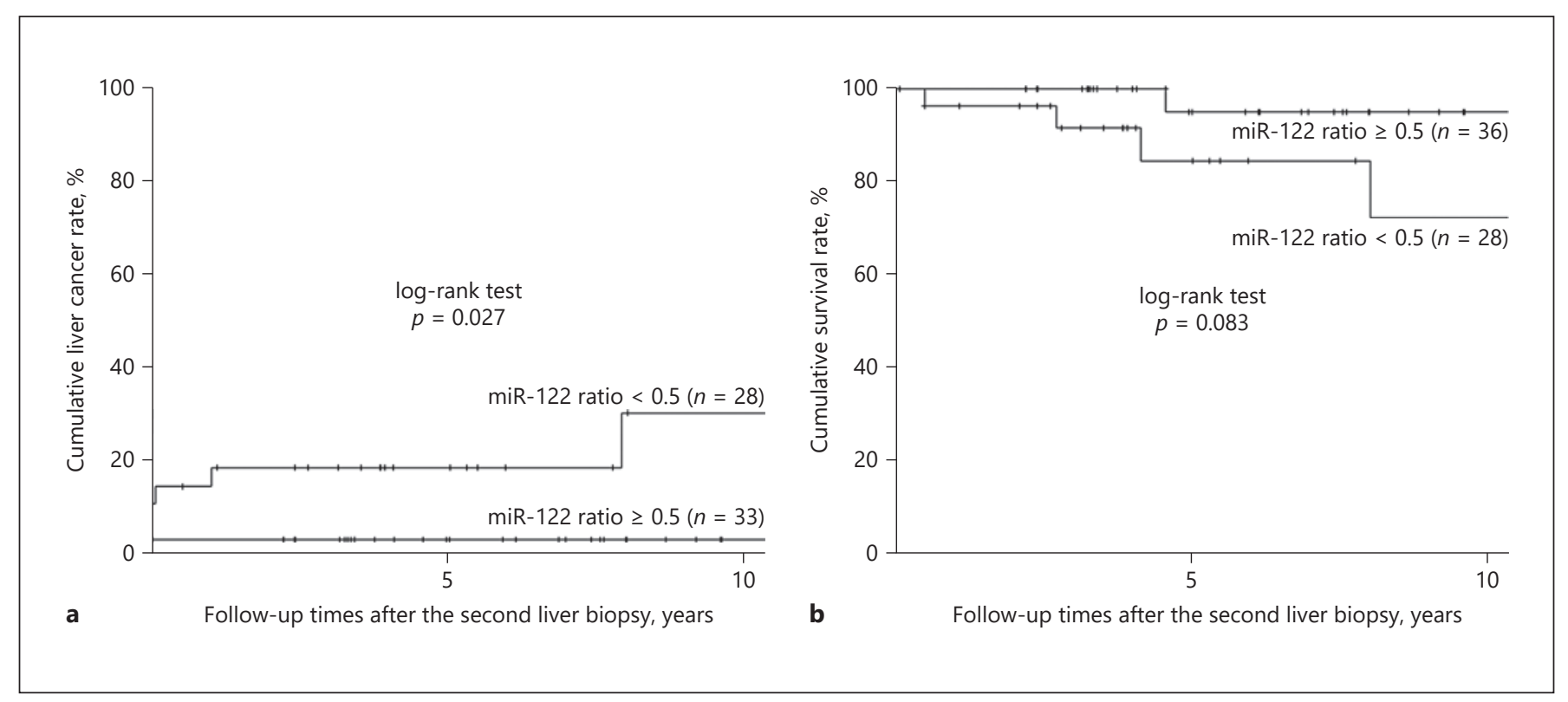

Fig. 3. Cumulative liver cancer and survival rates according to circulating miR-122 dynamics in patients without improvement of stage scores (high-risk group). a Cumulative liver cancer rates of those with miR-122 ratios $<0.5$ were significantly higher than those with ratios $\geq 0.5$ ( $p=0.007$; log-rank test). $\mathbf{b}$ Cumulative survival rates of those with miR-122 ratios $<0.5$ tended to be lower than those with ratios $\geq 0.5(p=0.083$; log-rank test). miR-122, microRNA-122.

\section{Cumulative Liver Cancer and Survival Rates}

According to miR-122 Ratio of High-Risk Group

During the median follow-up of 4.5 years after the second liver biopsy (range, $0.0-16.0$ years), there were 7 $(10.9 \%)$ cases of liver cancer and $5(7.8 \%)$ deaths in the high-risk group. In cases of liver cancer, the median interval between the second liver biopsy and incidence of liver cancer was 0.1 years (range, $0.0-7.8$ years). In cases of death, the median interval between the second liver biopsy and death was 4.1 years (range, $0.5-7.9$ years). The cumulative rates of liver cancer for those with miR-122 ratios $<0.5$ were significantly higher than those with ratios $\geq 0.5$ ( $p=0.007$; log-rank test), as shown in Figure 3a. The cumulative survival rates for those with miR-122 ratios $<0.5$ tended to be lower than those with ratios $\geq 0.5$ ( $p$ $=0.083$; log-rank test), as shown in Figure 3b.

Of the 64 patients who showed no improvement of the stage scores, 39 were in stage 2 or higher (severe fibrosis stage) at the first liver biopsy and underwent analyses to determine the cumulative liver cancer and survival rates according to the miR-122 ratio. During the median follow-up of 4.0 years after the second liver biopsy (range, $0.1-15.5$ years), there were $6(15.4 \%)$ cases of liver cancer and $5(12.8 \%)$ deaths. In cases of liver cancer, the median interval between the second liver biopsy and incidence of liver cancer was 0.1 years (range, $0.0-7.8$ years). In cases of death, the median interval between the second liver biopsy and death was 4.1 years (range, $0.5-7.9$ years). The cumulative rates of liver cancer for those with miR-122 ratios $<0.5$ were significantly higher than those with ratios $\geq 0.5$ ( $p=0.040$; log-rank test), and the cumulative survival rates for those with ratios $<0.5$ tended to be lower than those with ratios $\geq 0.5$ ( $p=0.070$; log-rank test). The results indicate that the miR-122 ratio was useful for the prediction of prognosis for those in the high-risk group (those who showed no improvement of stage scores), especially patients with stage 2 or higher at the first liver biopsy.

\section{Discussion}

It remains unclear what the impacts of epigenetic factors are on the mortality of NAFLD patients, including miRs. A recent review suggests that miRNAs have roles in the progression of NAFLD. miR-21 accelerates the progression of NAFLD, whereas miR-122 and miR223 ameliorate it. miR-21 and miR-122 control liver
Akuta et al. 
steatosis by regulating genes that are involved in the synthesis of triglyceride and its secretion. miR-122 attenuates liver inflammation and fibrosis by reducing the infiltration of inflammatory CD11bhiGr1+ cells, as well as targeting Ccl2 and Klf6 in hepatocytes. miR-21 and miR-155 act as onco-miRNAs in NAFLD-related liver cancer, whereas miR-122 and miR-223 protect against the development of HCC [20]. However, there is a lack of information on serial liver biopsies in regard to the long-term prognosis associated with circulating miR-122 dynamics in patients with histopathologically confirmed NAFLD.

Reports indicate that serum miR-122 is an independent predictor of overall survival for patients with liver cirrhosis [21] and liver cancer [22, 23]. However, these reports did not investigate the impact of miR-122 based on a longitudinal examination of serial liver biopsies from patients with NAFLD. Our previous report identified that there is a lack of information in regard to liver cancer or the histopathological severity of NASH as independent predictors of high levels of serum miR-122 [13]. Serum miR-122 levels tended to be low in cases of fibrosis stage 4 and demonstrated a biphasic change with progression of the fibrosis stage. A long-term follow-up study of 1 patient's development showed that serum miR-122 levels tended to decrease before the progression of fibrosis stage 4 and the development of liver cancer [13].

The present results add support to the previous study and confirm that the dynamics of circulating miR-122 is a factor in poor prognosis. To our knowledge, no other studies have used serial liver biopsies to investigate the relationships between miR-122 dynamics and prognosis, including liver cancer and mortality. Our results highlight the importance of circulating miR-122 dynamics for early diagnosis and treatment to improve the prognosis of patients with NAFLD. The limitations of present study are that several patients without HCC also indicated the decrease of miR-122 levels between first biopsy and second biopsy. Furthermore, the optimal interval of miR122 measurements could not be also concluded. Further studies, based on the suitable follow-up, should be performed to determine the usefulness of early diagnosis by combination with the other predictive factor for mortality and liver cancer development.

A meta-analysis of 11 studies involving 1,124 patients reported that low miR-122 expression in liver cancer tissues was significantly correlated with unfavorable overall survival in patients with liver cancer who underwent curative resection. However, the miR-122 expression level in the blood could not predict overall survival [24]. Thus, the expression profiles of tissue miR and circulating $\mathrm{miR}$ are not always consistent [25]. This discrepancy between the previous report and the present study could be due to differences in the methods used to measure miR-122 levels or in the etiology of liver cancer. One of the limitations of the present study is the lack of analysis of the miR-122 expression in tissues. Further studies should be performed with larger numbers of patients who have NAFLD to determine the effects on prognosis of the miR-122 expression in both the serum and liver tissue.

The present results support that miR-122 might protect against the development of liver cancer in patients with NAFLD. Furthermore, restoration of miR-122 might suppress the growth of liver cancer and render the cancer sensitive to chemotherapeutic agents [26, 27]. Large-scale prospective studies are needed to investigate the impact of miR-122 on the prognosis of patients with NAFLD, as well as to develop more effective therapeutic regimens for patients with NAFLD-related cirrhosis and liver cancer.

In conclusion, longitudinal examination of serial liver biopsies showed that the circulating miR-122 dynamics might be useful for predicting the prognosis of NAFLD patients with a severe stage of fibrosis who do not show improvement in the stage scores. Nevertheless, further studies should be performed with larger numbers of patients to determine the molecular mechanisms of the complex relationship between the impact of miR-122 on the epigenetic risk and pathogenesis of NAFLD.

\section{Acknowledgments}

The authors thank Drs. Keiichi Kinowaki and Takeshi Fujii (Department of Pathology, Toranomon Hospital) and Drs. Fukuo Kondo and Toshio Fukusato (Department of Pathology, Teikyo University School of Medicine) for their assistance in histopathological diagnosis.

\section{Statement of Ethics}

The study protocol was approved by the Human Ethics Review Committee at Toranomon Hospital (\#953, \#1135), and each patient provided a signed informed consent form at the time of liver histological diagnosis. The study was conducted in compliance with the International Conference on Harmonization Guidelines for Good Clinical Practice (E6) and the 2013 Declaration of Helsinki. 


\section{Conflict of Interest Statement}

(1) Hiromitsu Kumada has received honoraria from Gilead Sciences, AbbVie Inc., Eisai Co., Ltd., and Dainippon Sumitomo Pharma. (2) Yusuke Kawamura has received honoraria from Eisai Co., Ltd. All other authors declare that they have no conflicts of interest.

\section{Funding Sources}

This study was supported in part by a Grant-in-Aid from the Japan Agency for Medical Research and Development (JP21fk0210058,JP21fk0210065,JP21fk0210073, andJP21fk0210090).

\section{Author Contributions}

N.A., Y.K., F.S., S.S., Y.A., N.M., S.F., H.S., T.H., M.K. (Masahiro Kobayashi), M.K. (Mariko Kobayashi), Y.S., K.I., and H.K. contributed to this work. N.A., Y.K., and Y.A. analyzed the data. N.A. wrote the manuscript.

\section{Data Availability Statement}

The datasets generated or analyzed in the present study are available from the corresponding author on reasonable request.

\section{References}

1 Angulo P. Nonalcoholic fatty liver disease. N Engl J Med. 2002;346(16):1221-31.

2 Williams R. Global challenges in liver disease. Hepatology. 2006;44:521-6.

3 Torres DM, Harrison SA. Diagnosis and therapy of nonalcoholic steatohepatitis. Gastroenterology. 2008;134:1682-98.

4 Vuppalanchi R, Chalasani N. Nonalcoholic fatty liver disease and nonalcoholic steatohepatitis: selected practical issues in their evaluation and management. Hepatology. 2009;49:306-17.

5 Sumida Y, Nakajima A, Itoh Y. Limitations of liver biopsy and non-invasive diagnostic tests for the diagnosis of nonalcoholic fatty liver disease/nonalcoholic steatohepatitis. World J Gastroenterol. 2014;20:475-85.

6 Akuta N, Kawamura Y, Arase Y, Saitoh S, Fujiyama S, Sezaki H, et al. Hepatocellular carcinoma is the most common liver-related complication in patients with histopathologicallyconfirmed NAFLD in Japan. BMC Gastroenterol. 2018;18:165.

7 Kleiner DE, Brunt EM. Nonalcoholic fatty liver disease: pathologic patterns and biopsy evaluation in clinical research. Semin Liver Dis. 2012;32:3-13.

8 Younossi ZM, Stepanova M, Rafiq N, Makhlouf H, Younoszai Z, Agrawal R, et al. Pathologic criteria for nonalcoholic steatohepatitis: interprotocol agreement and ability to predict liver-related mortality. Hepatology. 2011;53:1874-82.

9 Angulo P, Kleiner DE, Dam-Larsen S, Adams LA, Bjornsson ES, Charatcharoenwitthaya P, et al. Liver fibrosis, but no other histologic features, is associated with long-term outcomes of patients with nonalcoholic fatty liver disease. Gastroenterology. 2015;149:38997.

10 Cermelli S, Ruggieri A, Marrero JA, Ioannou GN, Beretta L. Circulating microRNAs in patients with chronic hepatitis $\mathrm{C}$ and non-alcoholic fatty liver disease. PLoS One. 2011;6: e23937.
11 Pirola CJ, Fernández Gianotti T, Castaño GO, Mallardi P, San Martino J, Mora Gonzalez Lopez Ledesma M, et al. Circulating microRNA signature in non-alcoholic fatty liver disease: from serum non-coding RNAs to liver histology and disease pathogenesis. Gut. 2015;64: $800-12$.

12 Akuta N, Kawamura Y, Arase Y, Saitoh S, Fujiyama S, Sezaki H, et al. Circulating microRNA-122 and fibrosis stage predict mortality of Japanese patients with histopathologically confirmed NAFLD. Hepatol Commun. 2019; 4:66-76.

13 Akuta N, Kawamura Y, Suzuki F, Saitoh S, Arase Y, Kunimoto H, et al. Impact of circulating miR-122 for histological features and hepatocellular carcinoma of nonalcoholic fatty liver disease in Japan. Hepatol Int. 2016;10: 647-56.

14 Chalasani N, Younossi Z, Lavine JE, Charlton $\mathrm{M}$, Cusi K, Rinella $\mathrm{M}$, et al. The diagnosis and management of nonalcoholic fatty liver disease: practice guidance from the American Association for the Study of Liver Diseases. Hepatology. 2018;67:328-57.

15 Kleiner DE, Brunt EM, Van Natta M, Behling C, Contos MJ, Cummings OW, et al. Design and validation of a histological scoring system for nonalcoholic fatty liver disease. Hepatology. 2005;41:1313-21.

16 Brunt EM, Janney CG, Di Bisceglie AM, Neuschwander-Tetri BA, Bacon BR. Nonalcoholic steatohepatitis: a proposal for grading and staging the histological lesions. Am J Gastroenterol. 1999;94:2467-74.

17 Akuta N, Kawamura Y, Suzuki F, Saitoh S, Arase Y, Fujiyama S, et al. Analysis of association between circulating miR-122 and histopathological features of nonalcoholic fatty liver disease in patients free of hepatocellular carcinoma. BMC Gastroenterol. 2016;16:141.
18 Kroh EM, Parkin RK, Mitchell PS, Tewari M. Analysis of circulating microRNA biomarkers in plasma and serum using quantitative reverse transcription-PCR (qRT-PCR). Methods. 2010;50:298-301.

19 Yu S, Liu Y, Wang J, Guo Z, Zhang Q, Yu F, et al. Circulating microRNA profiles as potential biomarkers for diagnosis of papillary thyroid carcinoma. J Clin Endocrinol Metab. 2012;97:2084-92.

20 Wang X, He Y, Mackowiak B, Gao B. MicroRNAs as regulators, biomarkers and therapeutic targets in liver diseases. Gut. 2021;70(4): 784-95.

21 Waidmann O, Köberle V, Brunner F, Zeuzem S, Piiper A, Kronenberger B. Serum microRNA-122 predicts survival in patients with liver cirrhosis. PLoS One. 2012; 7:e45652.

22 Köberle V, Kronenberger B, Pleli T, Trojan J, Imelmann E, Peveling-Oberhag J, et al. Serum microRNA-1 and microRNA-122 are prognostic markers in patients with hepatocellular carcinoma. Eur J Cancer. 2013;49:3442-9.

$23 \mathrm{Xu} \mathrm{Y,} \mathrm{Bu} \mathrm{X,} \mathrm{Dai} \mathrm{C,} \mathrm{Shang} \mathrm{C.} \mathrm{High} \mathrm{serum} \mathrm{mi-}$ croRNA-122 level is independently associated with higher overall survival rate in hepatocellular carcinoma patients. Tumour Biol. 2015;36:4773-6.

24 Zhang Y, Li Y, Jiang W, Li Q, Lan Y. The clinical significance of microRNA-122 in predicting the prognosis of patients with hepatocellular carcinoma: a meta-analysis validated by the cancer genome atlas dataset. Medicine. 2019;98:e14810.

25 Morishita A, Masaki T. MicroRNAs as possible biomarkers for hepatocellular carcinoma. Hepatol Res. 2018;48:499-501.

26 Szabo G, Bala S. MicroRNAs in liver disease. Nat Rev Gastroenterol Hepatol. 2013;10(9): 542-52.

27 Bandiera S, Pfeffer S, Baumert TF, Zeisel MB. miR-122: a key factor and therapeutic target in liver disease. J Hepatol. 2015;62:448-57. 